\title{
Clinical impact of EUS elastography followed by contrast-enhanced EUS in patients with focal pancreatic masses and negative EUS- guided FNA
}

\author{
Sevastiţa Iordache', Mădălin Ionuţ Costache', Carmen Florina Popescu², Costin-Teodor Streba ${ }^{1}$, \\ Sergiu Cazacu ${ }^{1}$, Adrian Săftoiu ${ }^{1,3}$
}

${ }^{1}$ Gastroenterology Department, Research Center of Gastroenterology and Hepatology of Craiova, University of Medicine and Pharmacy Craiova, Romania, ${ }^{2}$ Cytology Laboratory, Pathology Department, Clinical Emergency County Hospital Craiova, Romania, ${ }^{3}$ Endoscopy Department, Gastrointestinal Unit, Copenhagen University Hospital Herlev, Denmark

\begin{abstract}
Aim: It is well known that endoscopic ultrasound guided fine needle aspiration (EUS-FNA) has a high sensitivity (over $85 \%)$ and specificity (100\%) for diagnosis of pancreatic cancer. The aim of the study was to establish a EUS based clinical diagnostic algorithm in patients with pancreatic masses and negative cytopathology after EUS-FNA, based on previously published results and cut-offs of real-time elastographic (RTE) EUS and contrast-enhanced harmonic (CEH) EUS. Material and methods: We included in the study a subgroup of 50 consecutive patients with focal pancreatic masses which underwent EUS examinations with negative EUS-FNA. RTE-EUS and CEH-EUS were performed sequentially in all patients. The sensitivity, specificity and accuracy of these methods were calculated separately. A clinical decision algorithm based on elastography followed by CEH was established. Results: For the diagnosis of possible malignancy, the sensitivity, specificity and accuracy of RTE-EUS were: $97.7 \%, 77.4 \%$, and $84 \%$ respectively. CEH-EUS had similar results: $89.5 \%, 80.7 \%$, and $84 \%$, respectively. In 25 patients with soft/mixed appearance during elastography,sequential assessment using contrast-enhanced EUSwas performed. The specificity of CEH-EUS for detection of chronic pancreatitis in this sub-set of patients was excellent (100\%). In other 25 patients with hard appearance in elastography (low strain) CEH-EUS had an excellent specificity (100\%) and accuracy (93\%) in the detection of pancreatic cancer. Conclusions: The proposed algorithm with sequential use of elastography followed by $\mathrm{CEH}$ could be a good clinical tool in the set of patients with negative EUS-FNA results for the differentiation between benign and malignant focal pancreatic masses.
\end{abstract}

Keywords: EUS, elastography, contrast enhancement, clinical algorithm, pancreatic masses

\section{Introduction}

Assessment of focal pancreatic masses is a challenge for gastroenterologists and endoscopists. Beyond imaging techniques such as multidetectorcomputer tomography (CT) scan and magnetic resonance imaging (MRI),

Received 28.09.2015 Accepted 15.11.2015

Med Ultrason

2016, Vol. 18, No 1, 18-24

Corresponding author: Adrian Saftoiu

Research Center of Gastroenterology and Hepatology

University of Medicine and Pharmacy

1 Mai Bvd, no 60

200355 Craiova, Dolj, Romania

Phone/Fax: +40251310.287

E-mail: adrian.saftoiu@umfcv.ro endoscopic ultrasound (EUS) offers more important data: tissue diagnosis. EUS-guided fine needle aspiration (FNA) seems to be the main technique used for the diagnosis of malignancy [1]. Nowadays, EUS-guided FNA represents the gold standard for the histological diagnosis of pancreatic malignancy. The overall accuracy of EUS with the addition of EUS-FNA is thus superior to the CT scan and MRI for detection and characterization of focal pancreatic lesions [2-5]. EUS also provides an excellent complement to multidetector CT for both diagnosis and staging of pancreatic cancer (PC) being able to detect small lesions (less than 1-2 cm) [6]. EUS also identifies patients unlikely to be cured by surgery due to vascular invasion or regional/distant nodal metastases [7]. Nevertheless, EUS-guided FNA is not perfect, 
reaching a sensitivity slightly over $85 \%$ and a specificity up to $100 \%$ for diagnosis of pancreatic adenocarcinoma [8-10]. Consequently, in a significant number of pancreatic adenocarcinoma patients have false negative results for EUS-FNA, especially in patients with concomitant chronic pancreatitis (CP). These delay the diagnosis decision as the patients cannot be referred directly to surgery or chemo-radiotherapy in the absence of a clear-cut tissue diagnosis of malignancy.

Real-time elastographic EUS (RTE-EUS) and contrast-enhanced harmonic EUS (CEH-EUS) are two useful methods for the assessment of patients with focal pancreatic masses both reported to have a high sensitivity and specificity [11-16]. The high negative predictive value of the combination between elastography and contrast enhancement during EUS as reported [16] has raised hopes that these imaging methods might help the clinical decision making the process of differentiation between benign and malignant focal pancreatic masses, especially in patients with negative EUS-FNA samples.

The aim of the study was to establish a EUS based clinical diagnostic algorithm in patients with focal pancreatic masses and negative cytology after EUS-FNA, by using elastography and contrast-enhancement during EUS examinations.

\section{Material and methods}

In this retrospective analysis of prospectively collected data we included 130 consecutive patients with focal pancreatic masses who underwent EUS with FNA during 4 years (2009-2012). From these patients, 50 patients (38.46\%) had negative FNA: 19 with pancreatic cancer (PC) and 31 with pseudotumoral chronic pancreatitis (CP). The inclusioncriteriawere patients with pancreatic masses assessed by EUS and age between $18-80$ years. The exclusion criteria were the presence of pancreatic neuroendocrine tumours, cystic mucinous tumors, or other malignancies.

The final diagnosis was established by repeated EUSFNA with positive smears, surgical resection (in operable patients), and follow-up during first year. In masses with a high suspicion of pancreatic cancer, EUS-FNA was repeated after 1 month. Elastography and contrastenhancement were performed sequentially in all patients during the same examination. EUS examination equipment included Hitachi 8500 or Hitachi Preirus ultrasound systems (Hitachi Medical Systems, Zug, Switzerland) with an embedded sonoelastography module, coupled with the EG 3830 linear endoscope (Pentax, Hamburg, Germany). The first assessment includes gray-scale imaging for detection and characterization of the focal mass followed by at least 3 passes of EUS-guided FNA.
The study was approved by the Ethical Committee of the University of Medicine and Pharmacy, Craiova, Romania. An informed consent was obtained from every patient. EUS and EUS-FNA procedures are performed routinely in the endoscopy unit during daily practice. Sonoelastography is a complementary procedure performed during EUS, being embedded in the US system analysis $[17,18]$. Contrast-enhanced US examinations were performed according to the protocol of EFSUMB guidelines [19]. The second-generation microbubble US contrast agent (SonoVue, Bracco Diagnostics Inc, Italy) used for contrast-enhanced analysis is approved for clinical use in Romania and the rest of the European Union countries.

\section{Real-time elastographic EUS}

Elastography information was displayed in a region of interest (ROI) which was manually selected and included the targeted lesion, as well as the soft surrounding tissues. The ROI needs to be set to include sufficient surrounding tissue because elasticity values are displayed relative to the average strain inside the ROI. To visualize tissue elasticity patterns, different elasticity values are marked with different colours (on a scale of 1 to 255) and the elastography information is shown superimposed on the conventional gray-scale image. The system is setup to use a hue colour map (red-green-blue), where hard tissue areas are marked with dark blue, medium hard tissue areas with cyan, intermediate tissue areas with green, medium soft tissue areas with yellow while soft tissue areas are red. Transducer compression is usually not necessary, because heart beats or respiratory movements replace manual compressions. In order to obtain semi-quantitative measurements we used hue (strain) histograms calculated on a ROI including only the tumour mass. Hence, the mean of the hue (strain) histogram values are equivalent to the mean elasticity values, while the standard deviation indicates the heterogeneity within the tumor mass. Cut-off values of the hue histogram over 175 characterized hard masses, as published previously, while this corresponds to a value of 80 for strain histograms which have a completely reversed scale from 0 to 255 [20,21].

\section{Contrast enhanced harmonic EUS}

An initial scan was performed by power Doppler EUS, followed by a bolus intravenous injection of 4.8 $\mathrm{mL}$ of SonoVue. Examinations during the early arterial phase (usually at 15-20 seconds) have been performed either in high mechanical index modes (with usual power Doppler), as well as low mechanical index mode with contrast harmonics. After 1 minute of continuous examination, the system has been switched back to power Doppler EUS. Consequently, tumours were considered iso-/ hypervascularvshypovascular as compared to surround- 


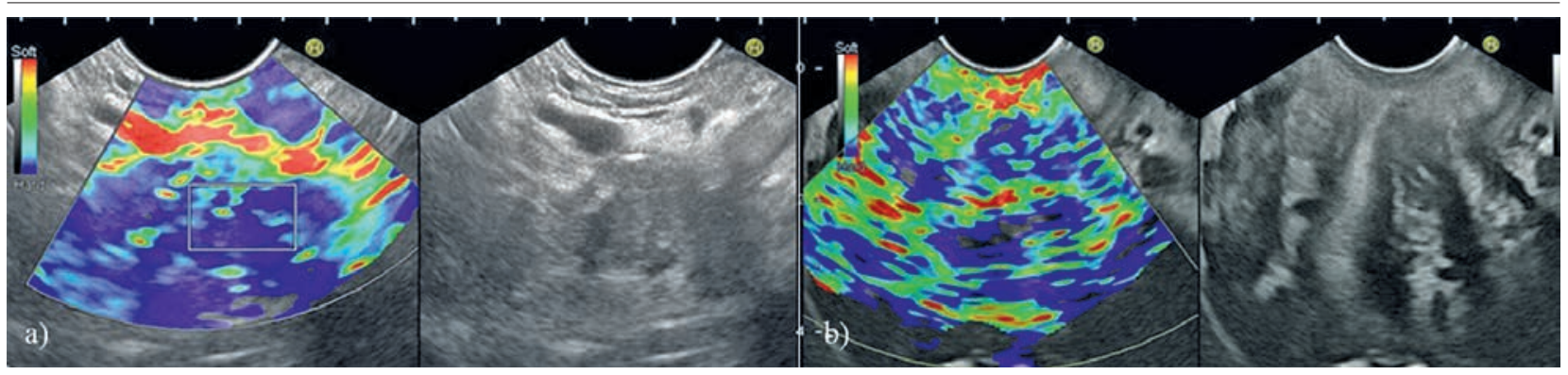

Fig 1. EUS elastography (qualitative assessment) in: a) Pancreatic cancer, hard (blue) pattern as compared with normal pancreatic tissue; b) chronic pseudotumoral pancreatitis with a mixed appearance (blue/green) inside the mass, similar with surrounding pancreatic tissue.

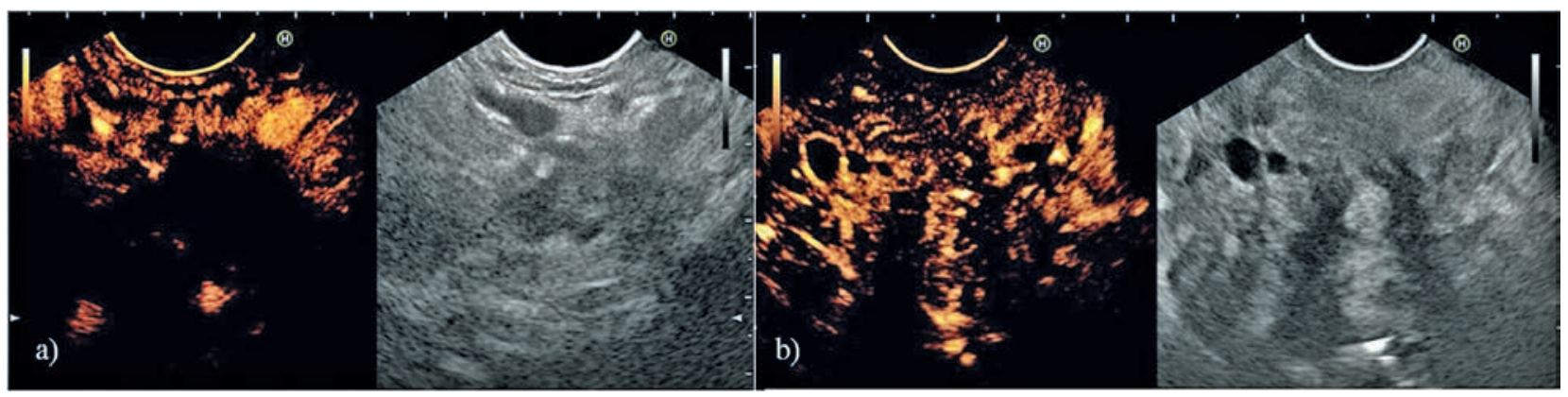

Fig 2. CEUS in a) Pancreatic cancer: hypovascular pattern in arterial and venous phases; b) chronic pancreatitis: hypervascularpattern in both arterial and venous phases.

Table I. Patients characteristicsand CEH-EUS and RTE-EUS findings

\begin{tabular}{llll}
\hline & $\begin{array}{l}\text { Chronic pancreatitis } \\
(\mathrm{n}=31)\end{array}$ & $\begin{array}{l}\text { Pancreatic cancer } \\
(\mathrm{n}=19)\end{array}$ & P value \\
\hline Age (y) & $50.58 \pm 11.41$ & $60.31 \pm 8.43$ & 0.0023 \\
Male/female & $31 / 0$ & $12 / 7$ & 0.0005 \\
Elastography (soft-mixed/hard) & $24 / 7$ & $1 / 18$ & $<0.0001$ \\
Elastography (hue histogram mean) & $155.03 \pm 23.81$ & $187.35 \pm 13.18$ & $<0.0001$ \\
Vascularisation (hyper/hypoenhanced) & $25 / 6$ & $17-$ Feb & $<0.0001$ \\
\hline
\end{tabular}

$\mathrm{n}=$ number of patiens; RTE-EUS - real-time elastographic endoscopic ultrasound; CEH-EUS - contrast-enhanced harmonic endoscopic ultrasound

ing pancreatic tissue (qualitative assessment). Moreover, quantitative assessment using a plug-in developed by IT Centre of University of Medicine and Pharmacy Craiova was possible, measuring the power Doppler vascularity index. The percent of colour pixels inside the tumor was thus calculated during 10 seconds video in the (late) venous phase of contrast enhancement [16]. For low mechanical index examinations, movies were also examined quantitatively with specialized quantification software (Vuebox, Bracco Diagnostics inc., Italy). This ensured a more objective analysis of tumour vascularity, as shown previously [22].

\section{Statistical analysis}

The standard deviation (SD) was used to quantify the amount of variation of continuous data values: age and hue histogram values in elastography.
The sensitivity, specificity, predictive positive value, negative positive value and accuracy were calculated separately for each method (RTE-EUS and CEH-EUS). Consecutively, these parameters were calculated for the combined methods. We selected two groups of patients with soft/mixed appearance and hard appearance in elastography both followed by CE-EUS assessment. These patients represent a category difficult to manage: surgical treatment versus extended follow-up. Thus, we considered it necessary to find an algorithm based on EUS parameters. This algorithm is based on assessment of pancreatic masses using elastographyfor characterization of hardness of the tissue followed by contrast-enhancement. Thus, the clinical decision making process could be variable according to both imaging methods, with some patients sent directly to surgery, others fol- 
Table II. The accuracy, sensitivity and specificity of CEH-EUS and RT-EUS

\begin{tabular}{lllll}
\hline & \multicolumn{3}{c}{ Type of tumors in RT-EUS and CEH-EUS } \\
& Soft+hypervascular & Hard+hypovascular \\
Results (\%) & $\mathbf{9 5 \%}$ CI & $53.28 \%$ to $90.16 \%$ & $88.89 \%$ & $65.29 \%$ to $98.62 \%$ \\
& $75.00 \%$ & $16.55 \%$ to $100.00 \%$ & $100.00 \%$ & $59.04 \%$ to $100.00 \%$ \\
Sensitivity & $100.00 \%$ & $2.37 \%$ to $57.77 \%$ & $77.78 \%$ & $39.99 \%$ to $97.19 \%$ \\
Specificity & $14.29 \%$ & $81.32 \%$ to $100.00 \%$ & $100.00 \%$ & $79.41 \%$ to $100.00 \%$ \\
Negative predictive value & $100.00 \%$ & & $92.00 \%$ & \\
Positive predictive value & $76.00 \%$ & & & \\
Accuracy & & & & CI $\%$ \\
\hline
\end{tabular}

RTE-EUS - real-time elastographic endoscopic ultrasound; CEH-EUS - contrast-enhanced harmonic endoscopic ultrasound

lowed-up and some to EUS-guided FNA. Analysis ofthe $2 \times 2$ contingency table was performed for categorical data withtwo-tailed $P$ value was obtained using Fisher's exact test while unpaired t-test was used for continuous data. A $P$ value below 0.05 was considered statistically significant.

\section{Results}

The study included 50 consecutive patients with focal pancreatic masses: 19 patients with pancreatic cancer and 31 patients with chronic pancreatitis, all of them with initial negative EUS-guided FNA. The mean age of the patients was 54.28 years $\pm 11.35,7$ females and 43 males. The characteristics of patients included in the study are listed in the Table I. The majority of tumours were located in the head of the pancreas (45 tumors), only 5 being in the body. In this set of patients with negative EUSguided FNA results there was no tumour located in the tail of the pancreas.

According to our results, the majority of pancreatic malignant tumors were hard to strain, with blue pattern in elastography while chronic pancreatitis had a soft or mixed appearance (fig 1).

The median value of the hue histogram in elastography was $166.79 \pm 25.75$. For pancreatic cancer the median value of elastography was significantly higher, as compared to chronic pseudotumoral pancreatitis $(p<0.001)$ (Table I). Qualitative assessment of contrast-enhancement classified masses in hypo-enhanced as the majority of malignant tumours or iso/hyper-enhanced as the majority of pseudotumoral chronic pancreatitis (fig 2). According to our results, this method had good results for the differentiation between malignant and benign pancreatic masses. The sensitivity, specificity and accuracy of RTE-EUS for the diagnosis of malignancy were: $94.73 \%$, $77.42 \%$ and $84 \%$, respectively. Likewise, CEH-EUS had similar results: $89.47 \%, 80.65 \%$ and $84 \%$, respectively.

Subsequently, we selected the 25 patients with soft/ mixed appearance during elastography for sequential assessment using contrast-enhanced EUS. The sensitiv-

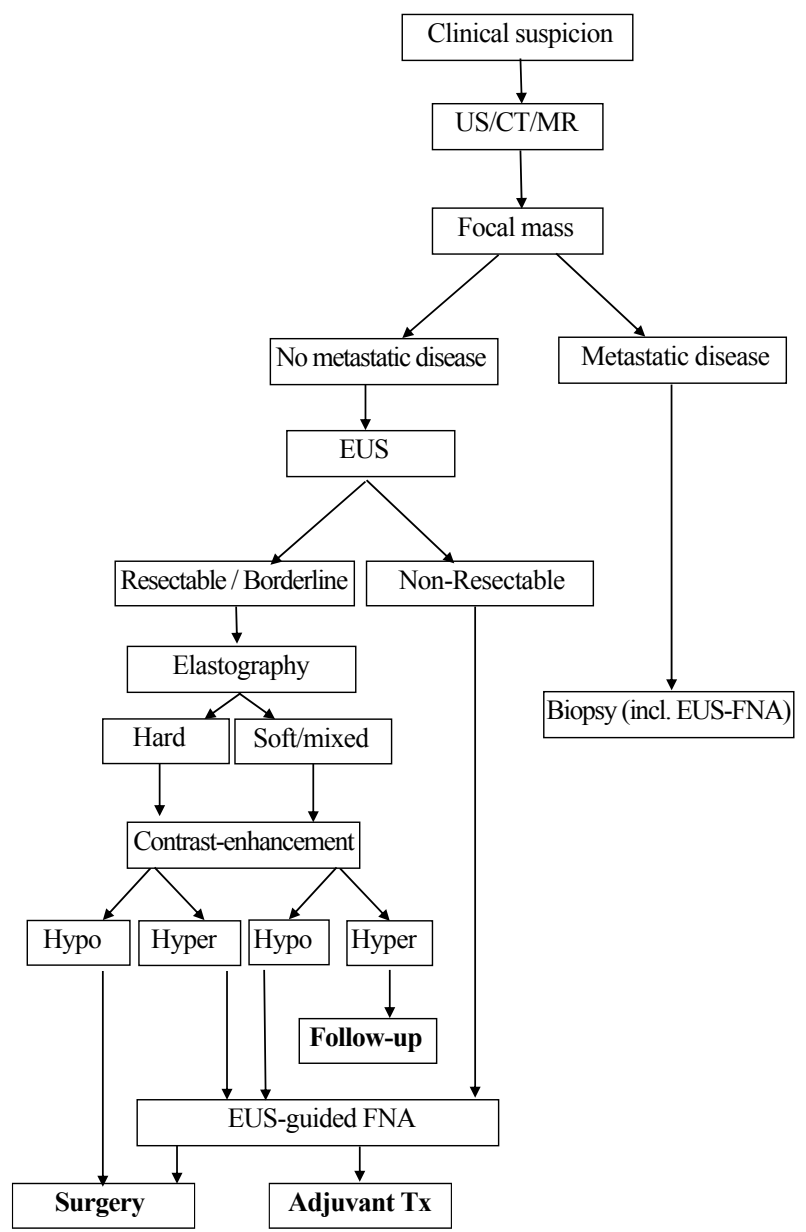

Fig 3. Clinical algorithm for assessment of patients with pancreatic masses

ity of CEH-EUS for the detection of chronic pancreatitis in this sub-set of patients was good $(75 \%)$, while the specificity was $100 \%$ with $76 \%$ accuracy. There were no false positive results, while the positive predictive value of this method was $100 \%$ (Table II). Moreover, unnecessary surgical resections can be avoided in patients with negative EUS-guided FNA characterized by mixed/soft appearance during RTE-EUS and iso/hypervascular pat- 
tern during CEH-EUS. These patients might be included in a follow-up program. On the other hand, there was another category: 25 patients with hard appearance in elastography (low strain). In these patients, CEH-EUS had an excellent specificity $(100 \%)$ and accuracy $(92 \%)$, while the sensitivity was $88.89 \%$ in the detection of pancreatic cancer. There are no cases with false positive results (Table II). Thus, this method allows the detection of patients that should be referred for surgery.

There is a minority of patients $(20 \%)$ that can be not clearly included in one of the 2 groups: patients with hard elastogram and hypervascular pattern and patients with soft/mixed elastogram and hypovascular pattern. These patients have to repeat EUS-FNA before making a decision. According to these results, we designed a clinical protocol for the sequential assessment of patients with pancreatic masses (fig 3).

\section{Discussions}

EUS and EUS-guided FNA are currently considered techniques of choice for the differential diagnosis of focal pancreatic masses. EUS has a high negative predictive value, reaching $100 \%$, virtually excluding pancreatic adenocarcinoma in patients with a normal pancreas during EUS. Meanwhile, EUS-FNA has a high specificity but a lower sensitivity in focal pancreatic masses. Whenever the suspicion of pancreatic adenocarcinoma is high, it is necessary to refer the patients directly to surgery or to repeat EUS-FNA. The current approach leads to increased morbidity and even mortality, because a significant number of patients with benign focal pancreatic masses are still operated on, without a clear cut preoperative diagnosis.

RTE-EUS and CEH-EUS have emerged as newer imaging techniques that can characterise the focal pancreatic masses in terms of strain (indirectly evaluating tissue hardness) or vascularity, respectively. Both techniques have an excellent accuracy, with a high sensitivity and low specificity reported in published meta-analyses [11-15]. RTE-EUS has a high sensitivity (95-100\%) but a lower specificity varying widely (22-74\%) [11-13]. According to previous reports, pancreatic cancer is hard to strain, with a blue pattern in elastography while chronic pancreatitis has a soft or mixed appearance. CEH-EUS represents a useful method to characterize vascular pattern of the pancreatic masses and hence to differentiate pancreatic adenocarcinomas from benign masses, with high sensitivity (up to 95\%) and specificity (90\%) [14]. Other studies were not so optimistic, indicating a sensitivity of $92 \%$ with a specificity of $68 \%$ [15]. Anyway, a hypovasculartumor is highly predictive for pancreatic adenocarcinoma $[14,15]$. Combined use of contrast-enhancement and elastography during the same EUS examination also has demonstrated a good sensitivity and specificity (75.8\%, 95.2\%, respectively) [16].Technically, they can be easily performed during EUS examinations, with a minimal prolongation of the examination time and virtually no complications, based on the excellent safety profile of microbubble ultrasound contrast agents.

Qualitative versus semi-quantitative RTE-EUS and CEH-EUS had similar values in our study, but also in the published meta-analyses $[11,12,14,15]$. Both can be used in clinical decision making algorithms, although a semiquantitative evaluation might yield more objective data used for subsequent analyses [21]. Recent advances in computer analysis and software systems might bring significant improvement and guidance during clinical procedures. Consequently, quantitative imaging biomarkers seem to steadily improve preclinical and clinical decision making [23]. Although this does not obviate the need for EUS-FNA it might certainly better select the patients and bring significant improvements in the methodology of EUS-FNA concerning the types of needle used, number of passes, cytology versus microhistology (core) biopsy, dedicated passes for ancillary studies, etc [24]. A recent published study revealed that CEH-EUS guided FNA has a better accuracy [25]. Moreover, fewer needle passes are required to obtain samples [26].

A combination of RTE-EUS and CEH-EUS might bring significant information for the differential diagnosis of focal pancreatic masses, as it significantly increases the specificity of the method [16]. Thus, hard (low-strain) hypovascular masses can be suggestive of pancreatic adenocarcinoma, while soft (high strain) iso/hypervascular masses can be suggestive of chronic pseudotumoral pancreatitis. This is extremely important in the setting of negative EUS-guided FNA, where the information obtained through advanced EUS techniques, could be used for the clinical decision making process. These two subgroups of patients can be clearly assigned for follow-up (based on the high NPV reaching 100\% for high strain, iso/hypervascular masses which are most probably benign) or surgery (based on the high PPV reaching up to $95 \%$ for low-strain, hypovascular masses which are most probable malignant). Intermediate subgroups can be further referred to repeat EUS-FNA, although they represented a minority of less than $20 \%$ of patients with negative EUS-FNA.Nevertheless, the results should be validated prospectively in a multicentric trial with a significant number of patients.

Limits of the study: Even the data were collected prospectively, the study is retrospective. There is a small number of patients included in this study as well. 


\section{Conclusion}

RTE-EUS thus represents an excellent method for differentiation between pancreatic adenocarcinoma from pseudotumoral chronic pancreatitis. Likewise, CEHEUS is a complementary method useful for characterization of focal pancreatic masses. The proposed algorithm with sequential use of elastography followed by contrastenhancement could be a good clinical tool in the set of patients with negative EUS-guided FNA results for the differentiation of benign and malignant focal pancreatic masses.

\section{Acknowledgements}

The study was partially supported by the research grant "Minimal invasive assessment of angiogenesis in pancreatic cancer based on imaging methods and molecular techniques (Angio-PAC)", Ideas programme, 164/2011, National Research Council - UEFISCDI, project number PN-II-ID-PCE-2011- 3-0589.

\section{Conflict of interest: none}

\section{References}

1. Hasan MK, Hawes RH. EUS-guided FNA of solid pancreas tumors. Gastrointest Endosc Clin N Am 2012; 22: 155-167.

2. Volmar KE, Vollmer RT, Jowell PS, Nelson RC, Xie HB. Pancreatic FNA in 1000 cases: a comparison of imaging modalities. Gastrointest Endosc 2005; 61: 854-861.

3. Gress FG, Hawes RH, Savides TJ, et al. Role of EUS in the preoperative staging of pancreatic cancer: a large singlecenter experience. Gastrointest Endosc 1999; 50: 786-791.

4. Will U, Mueller A, Topalidis T, Meyer F. Value of endoscopic ultrasonography-guided fine needle aspiration (FNA) in the diagnosis of neoplastic tumor(-like) pancreatic lesions in daily clinical practice. Ultraschall Med 2010; 31: 169-174.

5. Horwhat JD, Paulson EK, McGrath K, et al. A randomized comparison of EUS-guided FNA versus CT or US-guided FNA for the evaluation of pancreatic mass lesions. Gastrointest Endosc 2006; 63: 966-975.

6. Kinney T. Evidence-based imaging of pancreatic malignancies. Surg Clin North Am 2010; 90: 235-249.

7. Varadarajulu S, Eloubeidi MA. The role of endoscopic ultrasonography in the evaluation of pancreatico-biliary cancer. Gastrointest Endosc Clin N Am 2005; 15: 497-511.

8. Vilmann P, Săftoiu A, Hollerbach S, et al. Multicenter randomized controlled trial comparing the performance of 22 gauge versus 25 gauge EUS-FNA needles in solid masses. Scand J Gastroenterol 2013; 48: 877-883.

9. Hartwig W, Schneider L, Diener MK, Bergmann F, Büchler MW, Werner J. Preoperative tissue diagnosis for tumours of the pancreas. Br J Surg 2009; 96: 5-20.
10. Hewitt MJ, McPhail MJ, Possamai L, Dhar A, Vlavianos P, Monahan KJ. EUS-guided FNA for diagnosis of solid pancreatic neoplasms: a meta-analysis. Gastrointest Endosc 2012; 75: 319-331.

11. Mei M, Ni J, Liu D, Jin P, Sun L. EUS elastography for diagnosis of solid pancreatic masses: a meta-analysis. Gastrointest Endosc 2013; 77: 578-589.

12. Xu W, Shi J, Li X, Zeng X, Lin Y. Endoscopic ultrasound elastography for differentiation of benign and malignant pancreatic masses: a systemic review and meta-analysis. Eur J Gastroenterol Hepatol 2013; 25: 218-224.

13. Dawwas MF, Taha H, Leeds JS, Nayar MK, Oppong KW. Diagnostic accuracy of quantitative EUS elastography for discriminating malignant from benign solid pancreatic masses: a prospective, single-center study. Gastrointest Endosc 2012; 76: 953-961.

14. Gong TT, Hu DM, Zhu Q. Contrast-enhanced EUS for differential diagnosis of pancreatic mass lesions: a meta-analysis. Gastrointest Endosc 2012; 76: 301-309.

15. Park JS, Kim HK, Bang BW, Kim SG, Jeong S, Lee DH. Effectiveness of contrast-enhanced harmonic endoscopic ultrasound for the evaluation of solid pancreatic masses. World J Gastroenterol 2014; 20: 518-524.

16. Săftoiu A, Iordache S, Gheonea DI, et al. Combined contrast-enhanced power Doppler and real-time sonoelastography performed during EUS, used in the differential diagnosis of focal pancreatic masses (with videos). Gastrointest Endosc 2010; 72: 739-747.

17. Bamber J, Cosgrove D, Dietrich CF, et al. EFSUMB guidelines and recommendations on the clinical use of ultrasound elastography. Part 1: Basic principles and technology. U1traschall Med 2013; 34: 169-184.

18. Cosgrove D, Piscaglia F, Bamber J, et al. EFSUMB guidelines and recommendations on the clinical use of ultrasound elastography. Part 2: Clinical applications. Ultraschall Med 2013; 34: 238-253.

19. Piscaglia F, Nolsøe C, Dietrich C F, et al. The EFSUMB Guidelines and Recommendations on the Clinical Practice of Contrast Enhanced Ultrasound (CEUS): Update 2011 on non-hepatic applications. Ultraschall Med 2012; 33: 33-59.

20. Săftoiu A, Vilmann P, Gorunescu F, et al; European EUS Elastography Multicentric Study Group. Accuracy of endoscopic ultrasound elastography used for differential diagnosis of focal pancreatic masses: a multicenter study. Endoscopy 2011; 43: 596-603.

21. Săftoiu A, Vilmann P. Differential diagnosis of focal pancreatic masses by semiquantitative EUS elastography: between strain ratios and strain histograms. Gastrointest Endosc 2013; 78: 188-189.

22. Gheonea DI, Streba CT, Ciurea T, Săftoiu A. Quantitative low mechanical index contrast-enhanced endoscopic ultrasound for the differential diagnosis of chronic pseudotumoral pancreatitis and pancreatic cancer. BMC Gastroenterol 2013; 13: 2 .

23. Prescott JW. Quantitative imaging biomarkers: the application of advanced image processing and analysis to clinical 
and preclinical decision making. J Digit Imaging 2013; 26: 97-108.

24. Bang JY, Ramesh J, Trevino J, Eloubeidi MA, Varadarajulu S. Objective assessment of an algorithmic approach to EUS-guided FNA and interventions. Gastrointest Endosc 2013; 77: 739-744.

25. Seicean A, Badea R, Moldovan-Pop A, et al. Harmonic Contrast-Enhanced Endoscopic Ultrasonography for the
Guidance of Fine-Needle Aspiration in Solid Pancreatic Masses. Ultraschall Med 2015. doi: 10.1055/s-00351553496

26. Sugimoto M, Takagi T, Hikichi T, et al. Conventional versus contrast-enhanced harmonic endoscopic ultrasonographyguided fine-needle aspiration for diagnosis of solid pancreatic lesions: A prospective randomized trial. Pancreatology 2015; 15: 538-541. 